\title{
The Effects of Carbendazim on Acute Toxicity, Development, and Reproduction in Caenorhabditis elegans
}

\author{
Jie Li $\mathbb{D}^{1},{ }^{1}$ Xinghua Zhou $\mathbb{D}^{1},{ }^{1}$ Caiqin Zhang, ${ }^{1}$ Yansheng Zhao, ${ }^{1}$ Ying $\mathrm{Zhu}^{1}{ }^{1}$ Jiayan Zhang, \\ Juan Bai, ${ }^{1}$ and Xiang Xiao ${ }^{1,2}$ \\ ${ }^{1}$ School of Food and Biological Engineering, Jiangsu University, Zhenjiang 212013, China \\ ${ }^{2}$ Jiangsu Jiangnan Biotech Co., Ltd., Zhanjiang 212300, China \\ Correspondence should be addressed to Xinghua Zhou; zxh2012@ujs.edu.cn
}

Received 20 May 2020; Revised 29 May 2020; Accepted 10 August 2020; Published 28 August 2020

Academic Editor: quancai sun

Copyright ( $\odot 2020$ Jie Li et al. This is an open access article distributed under the Creative Commons Attribution License, which permits unrestricted use, distribution, and reproduction in any medium, provided the original work is properly cited.

Carbendazim, as a fungicide, was commonly used to control fungal diseases in agriculture, forestry, and veterinary medicines. In this study, the acute and reproductive toxicity of carbendazim was assessed using Caenorhabditis elegans (C. elegans) as a model in order to preliminarily evaluate the potential risks of this fungicide in agricultural production and application. The results showed that the growth of C. elegans was inhibited by $0.01 \mu \mathrm{g} / \mathrm{L}$ carbendazim. The treatment of $0.1 \mu \mathrm{g} / \mathrm{L}$ carbendazim caused a significant decrease in locomotion behavior and significant damage to the reproductive and antioxidant system, causing the lifespan of nematodes to be drastically shortened. These results provide a better understanding of the environmental risk of carbendazim and raise new concerns about safety.

\section{Introduction}

Pesticides, a kind of chemical or biological reagents, are widely used in agriculture to regulate plant growth and control diseases and insect pests, which can promote crop growth and improve crop yield [1]. However, the widespread use of pesticides will lead to different degrees of residues in crops or food and thus affect human health [2]. The problems of pesticide residues have not only attracted great attention of consumers but also become one of the key factors affecting food safety [3].

Carbendazim, as a broad-spectrum fungicide, has been used to control fungal diseases in agriculture, forestry, and veterinary medicines [4]. However, carbendazim is categorized in the hazardous category of chemicals by the World Health Organization [5] and has been classified in the priority list of endocrine-disrupting chemicals by the European Commission [6]. In recent years, it is obvious that the widespread use of carbendazim with over-range and overdose and the fact that carbendazim is hard to be degraded both lead to the problem of carbendazim residues in agriculture [7]. Although the toxic effect of carbendazim has been reported since the 1980s, the toxicity of carbendazim becomes a hot topic because of the increasing concern about environmental endocrine disruptors [4]. Carbendazim has been banned in several countries because of its negative impacts on the environment and health such as development and reproductive disturbances, toxicity, and mutagenicity [8]. The adverse effects of carbendazim on the biochemical, histopathological, and hematological parameters in the liver, kidney, and endocrine glands and their hormonal levels have been illustrated in rats [4]. Additionally, it needs further study on low concentration due to the residues of carbendazim.

Caenorhabditis elegans (C. elegans), as an important research model, is widely used to do some assessment. According to Amrit et al. [9], C. elegans has many advantages, such as small size, rapid generation time, easy of culturing on laboratory, and short adult lifespan. C. elegans was chosen in this study as the model organism to evaluate the toxicity of low concentration of carbendazim, which may be considered as a reference value for the application of carbendazim in agriculture. 


\section{Materials and Methods}

2.1. Chemicals and Strains. Carbendazim (purity $\geq 99 \%$; Aladdin ${ }^{\circledR}$ Biochemical Technology Co., LTD, Shanghai, China) was dissolved in N, N-dimethylformamide (DMF; Sinopharm Chemical Reagent Co., LTD, Shanghai, China) to produce $1 \mathrm{~g} / \mathrm{L}$ carbendazim original solution. The concentrations of DMF were $0.1 \%$ in final exposure solutions (0.01, 0.1, 1, 10, $100 \mu \mathrm{g} / \mathrm{L}) .0 .1 \%$ DMF without carbendazim was the control group. C. elegans (wild-type N2) were originally obtained from the Caenorhabditis Genetics Center (University of Minnesota, MN, USA). The nematodes were cultivated on nematode growth medium (NGM) plates that were seeded with Escherichia coli OP50 at $20^{\circ} \mathrm{C}$ as described [10]. L1-larval C. elegans were collected by washing the gravid nematodes with a bleaching mixture (1 M NaOH, $10 \% \mathrm{NaHOCl})$.

2.2. Lethality. Carbendazim original solutions $(1 \mathrm{~g} / \mathrm{L})$ were diluted with $\mathrm{S}$ liquid medium $\left(1.12 \mathrm{~g} \mathrm{~K} \mathrm{~K}_{2} \mathrm{HPO}_{4}, 5.92 \mathrm{~g}\right.$ $\mathrm{KH}_{2} \mathrm{PO}_{4}$, and $5.85 \mathrm{~g} \mathrm{NaCl}$ were diluted with $1 \mathrm{~L}$ water) to get the final carbendazim concentrations of $0,0.2,0.4,0.6,0.8$, and $1 \mathrm{mg} / \mathrm{L}$, which contained $0.1 \%$ DMF. S liquid medium with $0.1 \%$ DMF was the control group. 30 nematodes (L4) were tested in 96-well plates for each concentration. The survival of nematodes was counted under a microscope after culturing for 24 hours in the incubator. The process of the test is based on the method of Xiang et al. [11]. Three parallel experiments were needed.

2.3. Locomotion Behavior. At least 10 C. elegans (L4) were picked randomly from each concentration to determine the locomotive behavior, which was recorded by both the headthrash frequency and the body bend times [12]. The number of head-thrash frequency was counted by changes in the direction of bending at the midbody of C. elegans in $1 \mathrm{~min}$. Measurement of body bend was defined as the times of the direction changes of the part of the nematodes cultured in NGM without E. coli OP50 in the $30 \mathrm{~s}$.

2.4. Growth and Development Assays. C. elegans exposed to carbendazim for $24 \mathrm{~h}$ was analyzed. The body length of nematodes exposed to carbendazim was assessed by the Image J software. The offspring of each C. elegans from L4 larvae to day 1 was recorded at the L3 stage after individually transferring to a new plate every day until reproduction ceased [13]. At least three parallel tests were carried out.

2.5. Lifespan Analysis. All of C. elegans tested for lifespan were cultured in the same condition at $20^{\circ} \mathrm{C}$. The synchronized C. elegans were cultivated in NGM plates with different concentrations of carbendazim until day 4. The tested nematodes then would be transferred into new NGM plates every 2 days. Surviving and dead C. elegans were recorded daily (beginning on the first day of adulthood) until all nematodes for each concentration had died [11]. At least three parallel tests were carried out.
2.6. Determination of Oxidative Damage. Intracellular ROS was measured with $2^{\prime}, 7^{\prime}$-dichlorodihydrofluoroscein diacetate $\left(\mathrm{H}_{2} \mathrm{DCFH}-\mathrm{DA}\right)$, which is the most common and sensitive reactive oxygen detection probe by far. The wildtype N2 C. elegans were washed in M9 buffer and then ultrasonically disrupted. The supernatant was analyzed the ROS level following the instruction of the ROS kit. The final working concentration of $\mathrm{H}_{2} \mathrm{DCHE}-\mathrm{DA}$ was $10 \mu \mathrm{M}$ [11]. The excitation and emission absorbance wavelengths were $485 \mathrm{~nm}$ and $535 \mathrm{~nm}$, respectively. At least three parallel tests were carried out.

Intracellular total superoxide dismutase (T-SOD) was determined according to the instruction of the T-SOD kit purchased from the Nanjing Jiancheng Bioengineering Institute. After washed with M9 three times, the examined nematodes were ultrasonically disrupted and reacted with a T-SOD kit. The absorbance wavelength was $550 \mathrm{~nm}$. In addition, the supernatant was used to detect the level of protein for each concentration, in which absorbance wavelength was $595 \mathrm{~nm}$. At least three parallel tests were carried out.

2.7. Data Analysis. All data were given as mean \pm standard error of the mean (SEM) by using one-way ANOVA. Graphs were presented using Origin 8.5 and GraphPad Primer 7, and statistical analysis was performed using the SPSS 19.0 software. The statistical significance level was conducted using ${ }^{*} p<0.05$ and ${ }^{* *} p<0.01$.

\section{Results}

3.1. Determination of the Locomotion Behavior of C. elegans after Carbendazim Acute Exposures. $\mathrm{LC}_{50} \mathrm{C}$. elegans were exposed to carbendazim for 24 hours to assess its acute toxic effects. Data are represented as shown in Table 1, and the obtained linear fitting equation was $y=2.180 x-0.223$ through data analysis. The obtained $\mathrm{LC}_{50}$ is $0.867 \mathrm{mg} / \mathrm{L}$.

Next, we assayed the determination of the locomotive behavior of $C$. elegans after carbendazim acute exposures by analyzing the data about head-thrash frequency and bodybending times of nematodes (Figures 1(a) and 1(b)). Both of them showed significant decreases at the carbendazim concentrations ranging from $0.01 \mu \mathrm{g} / \mathrm{L}$ to $100 \mu \mathrm{g} / \mathrm{L}$ $(p<0.01)$. Additionally, the head thrashes of nematodes exposed to $100 \mu \mathrm{g} / \mathrm{L}$ decreased to $68.27 \%$. For the body bends test, when the carbendazim concentrations were $10 \mu \mathrm{g} / \mathrm{L}$ and $100 \mu \mathrm{g} / \mathrm{L}$, it had a significant inhibitory effect on the body bends of C. elegans by $36.77 \%$ and $35.48 \%$ compared with the control one, respectively.

3.2. Determination of the Growth and Development of C. elegans after Carbendazim Acute Exposures. Compared with the control group (Figures 1(c) and 1(d)), body length and body surface area were significantly $(p<0.01)$ reduced in the exposure groups from $0.01 \mu \mathrm{g} / \mathrm{L}$ to $100 \mu \mathrm{g} / \mathrm{L}$. Both of them were decreased by $19.16 \%$ and $22.15 \%$ at the treatment of $0.01 \mu \mathrm{g} / \mathrm{L}$ compared with the control group, respectively. The concentration of $10 \mu \mathrm{g} / \mathrm{L}$ presented the most negative 
TABLE 1: Effects of carbendazim on $\mathrm{LC}_{50}$ C. elegans by 24-h acute exposures.

\begin{tabular}{lcc}
\hline Concentration $(\mathrm{mg} / \mathrm{L})$ & Total & Survival \\
\hline 0 & 30 & 28 \\
0.2 & 30 & 25 \\
0.4 & 30 & 21 \\
0.6 & 30 & 18 \\
0.8 & 30 & 16 \\
1.0 & 30 & 15 \\
\hline
\end{tabular}

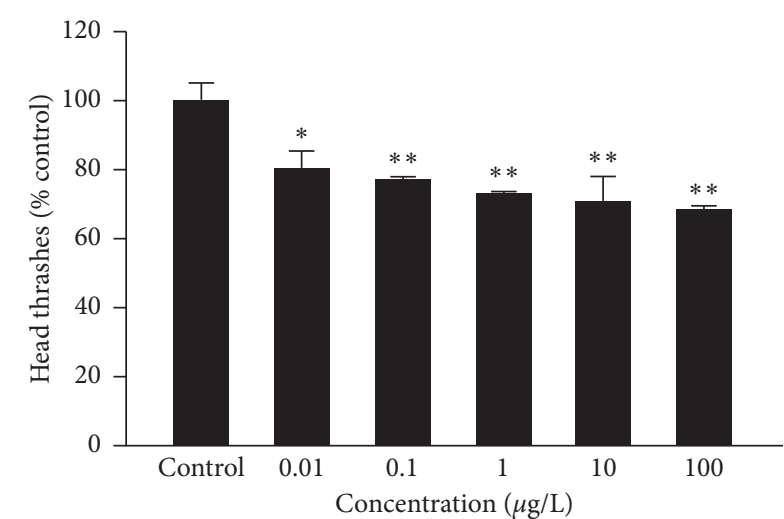

Carbendazim

(a)

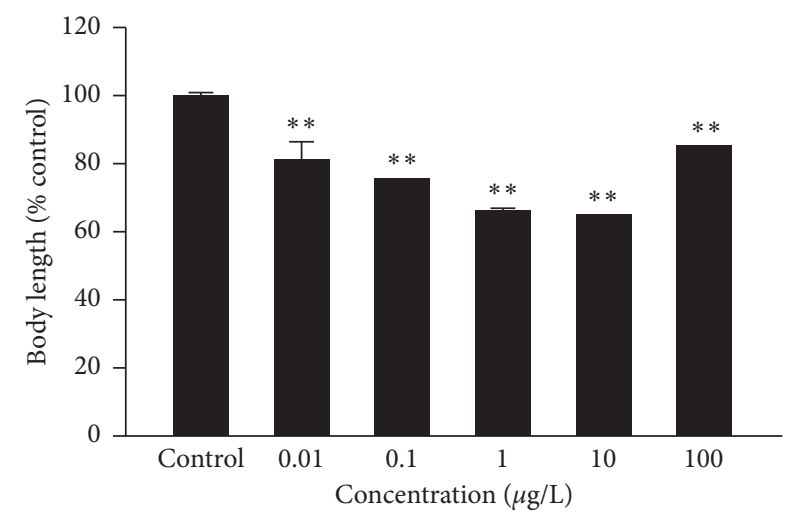

Carbendazim

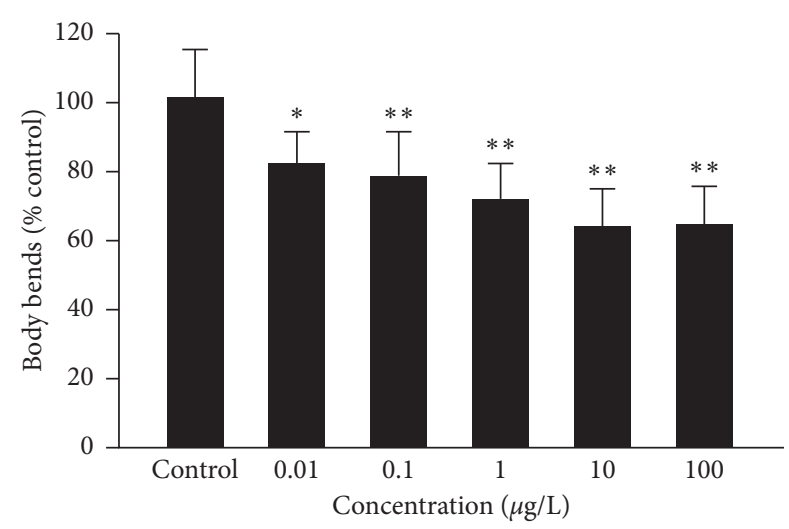

Carbendazim

(b)

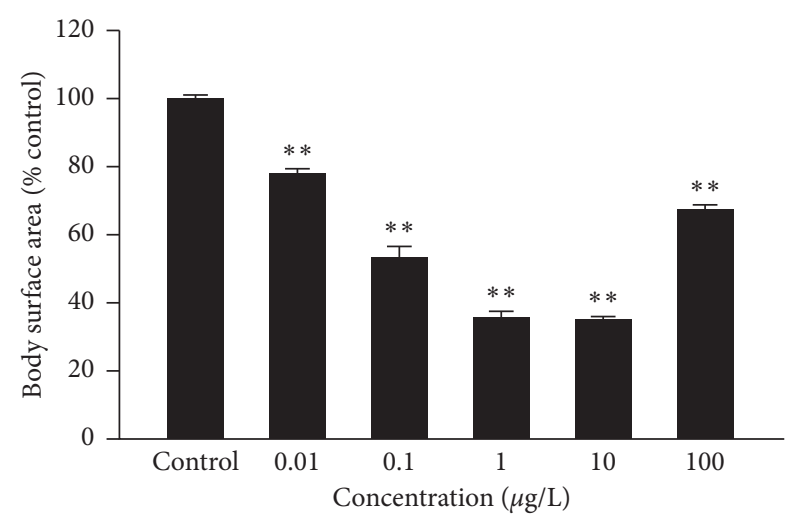

Carbendazim

(c)

(d)

FIGURE 1: Effects of C. elegans on physiological traits exposed to carbendazim. (a) The head thrashes of C. elegans after carbendazim exposure; (b) the body bends of $C$. elegans after carbendazim exposure; (c) the body length of C. elegans after carbendazim exposure; and (d) the body surface area of C. elegans after carbendazim exposure. Data (mean \pm SEM) are shown in figures as the percentage values compared with the control group. The asterisks present the significances between the each exposure group and control group $\left(^{*} p<0.05\right.$ and $\left.{ }^{* *} p<0.01\right)$.

impacts, and body length and body surface area of C. elegans were decreased by $35.21 \%$ and $65.22 \%$ compared with the control group, respectively.

3.3. Determination of the Brood Sizes of C. elegans after Carbendazim Acute Exposures. According to Figure 2, brood sizes of nematodes had a significant decrease $(p<0.01)$ in the treatment groups from $0.1 \mu \mathrm{g} / \mathrm{L}$ to $100 \mu \mathrm{g} / \mathrm{L}$. The brood sizes of $C$. elegans decreased most significantly, which decreased to $43.71 \%$ with the treatment of $10 \mu \mathrm{g} / \mathrm{L}$ compared with the control group.

3.4. Determination of the Lifespan of C. elegans after Carbendazim Acute Exposures. C. elegans lifespan was significantly inhibited by $0.01 \mu \mathrm{g} / \mathrm{L}$ to $100 \mu \mathrm{g} / \mathrm{L}$ carbendazim according to the lifespan curve shown in Figure 3. The results presented that $C$. elegans lifespan was decreased from 24 to 20 days with the treatment of $0.01 \mu \mathrm{g} / \mathrm{L}$ carbendazim. The 


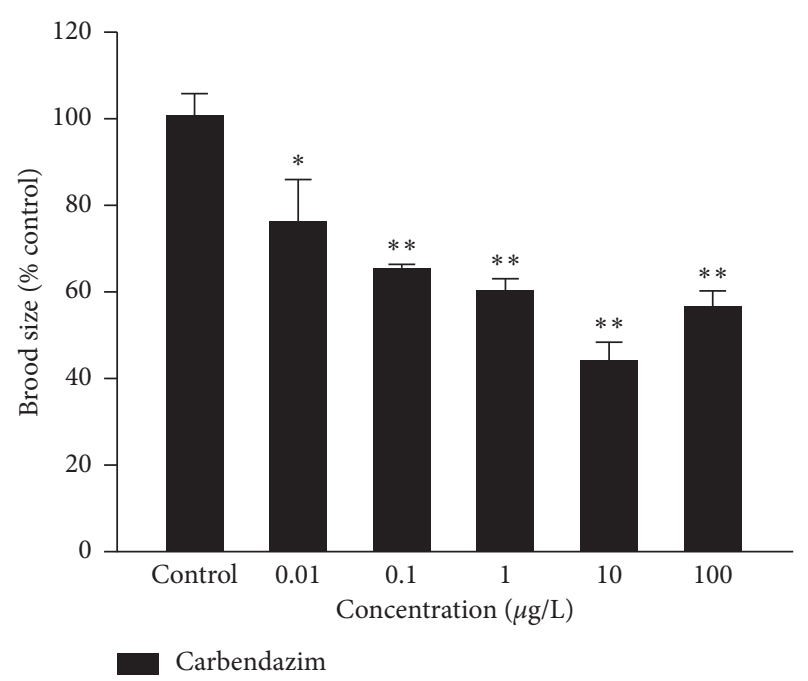

Figure 2: Effects of C. elegans on brood size exposed to carbendazim. Data (mean \pm SEM) are shown as the percentage value compared with the control group. The asterisks present the significance between the each exposure group and control group $\left({ }^{*} p<0.05\right.$ and $\left.{ }^{* *} p<0.01\right)$.

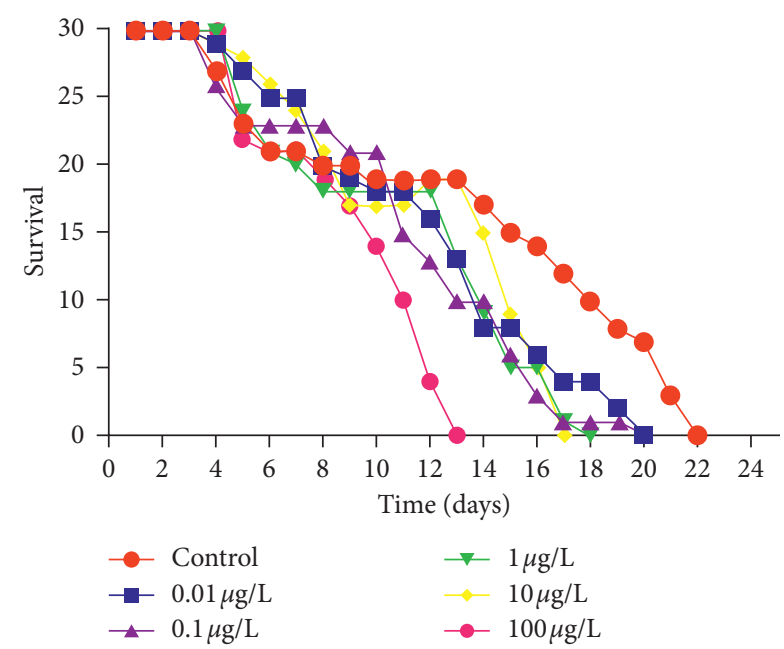

Figure 3: Effects of C. elegans on survival exposed to carbendazim. Data (mean \pm SEM) are shown as the percentage value compared with the control group. The asterisks present the significance between the each exposure group and control group $\left({ }^{*} p<0.05\right.$ and ** $p<0.01)$.

lifespan of nematodes treated with $0.01 \mu \mathrm{g} / \mathrm{L}$ carbendazim was reduced by $20.00 \%$. When the carbendazim exposure concentration was $100 \mu \mathrm{g} / \mathrm{L}$, the lifespan of C. elegans was reduced the most by $45.83 \%$.

3.5. Effects of Acute Exposure to Carbendazim on the Antioxidant System of C. elegans. The ROS levels of control and treated $C$. elegans at carbendazim exposure of different concentrations are shown in Figure 4(a). It was indicated that the level of intracellular ROS was significantly increased $(p<0.01)$ at ranges from $0.01 \mu \mathrm{g} / \mathrm{L}$ to $100 \mu \mathrm{g} / \mathrm{L}$ carbendazim. Compared with the control one, the ROS level was increased at most by $70.60 \%$ with the treatment of $10 \mu \mathrm{g} / \mathrm{L}$. According to the results of intracellular SOD levels, it had an increase at the treatment from $0.01 \mu \mathrm{g} / \mathrm{L}$ to $100 \mu \mathrm{g} / \mathrm{L}$ carbendazim (Figure 4(b)). The SOD level was increased by $10.70 \%$ at $0.1 \mu \mathrm{g} / \mathrm{L}$ carbendazim compared with the control group.

\section{Discussion}

Carbendazim, as the fungicides, is widely used in agriculture to inhibit the growth of fungus. Carbendazim has been prohibited to use in Australia, most of the European Union, and the USA because of its severe toxicology and persistent nature [14]. For this study, it was the first time to use C. elegans as the model organisms to evaluate the effects of carbendazim on locomotive behavior, growth and development, reproduction, lifespan, and antioxidant systems. Moreover, results showed that it had a negative influence on C. elegans.

According to the $24 \mathrm{~h}-\mathrm{LC}_{50}$, the acute toxicity concentration of C. elegans exposed to different concentrations of carbendazim is $0.867 \mathrm{mg} / \mathrm{L}$. The $96-\mathrm{h} \mathrm{LC}_{50}$ of carbendazim in response to zebrafish has been illustrated as $1.75 \mathrm{mg} / \mathrm{L} \mathrm{[15].} \mathrm{The}$ eggs of Prussian carp Carassius gibelio has shown the toxicity effects at the concentration of $0.036 \mathrm{mg} / \mathrm{L}[16,17]$. Studies presented that the growth and development of Navicula sp. is inhibited by carbendazim with a $24 \mathrm{~h}-\mathrm{EC}_{50}$ value of $2.18 \mathrm{mg} / \mathrm{L}$. Though the rate of algal growth is recovered after 72 -h exposure, the chlorophyll-a content remains significantly decreased when the treatment of carbendazim was beyond $0.5 \mathrm{mg} / \mathrm{L}$ [18]. In this present, C. elegans exposed to a low concentration of carbendazim were selected to evaluate its effects depending on the actual concentration of human daily exposure. Low concentrations of carbendazim do not mean it is safe. Carbendazim shows negative biological impacts at much lower doses in some studies.

Locomotive behavior was evaluated to assess the neurotoxicity of C. elegans (L4 larva) after 24-h exposure to carbendazim. The results showed that carbendazim could have negative effects on locomotive behavior through the detection of head thrashing and body bending of C. elegans, which both were more sensitive at the higher exposure group. The locomotive behavior of zebrafish embryos exposed to carbendazim is sensitive [15]. Previous studies have shown that fish have an abnormal behavior when sublethal concentrations of carbendazim are $0.22-0.43 \mathrm{mg} / \mathrm{L}$ [19].

Developmental malformations could also be one reason for abnormal locomotion [20]. The growth and development of C. elegans were assayed in our study. Results showed that the body length and body area of C. elegans were significantly narrowed at the treatment exceeding $0.01 \mu \mathrm{g} / \mathrm{L}$ carbendazim. The normal growth of vertebrates is related to the metabolic thyroid hormone homeostasis [21, 22]. Williams et al. [23] have indicated that carbendazim could cause sperm loss after implantation, fetal malformation, and slow growth and development.

The reproductive toxicity of carbendazim has been demonstrated that carbendazim could inhibit the microtubule polymerization of fungal and mammalian cells, causing disruption of microtubule assembly by acting with 


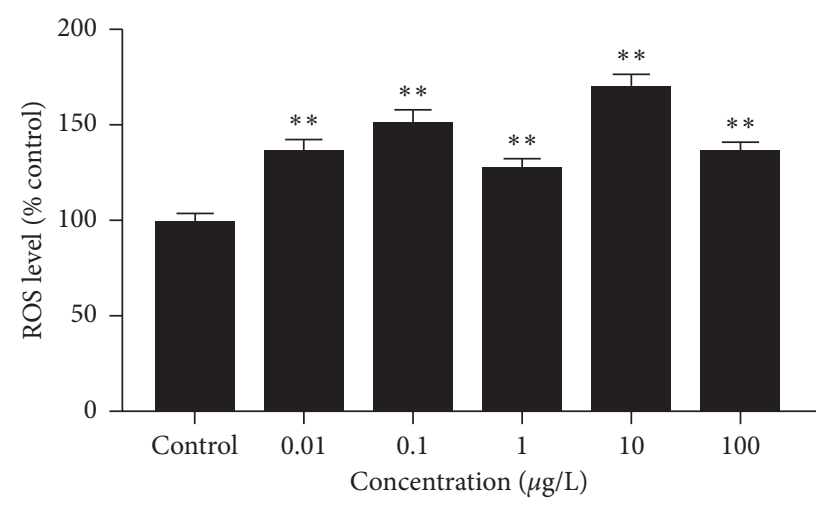

Carbendazim

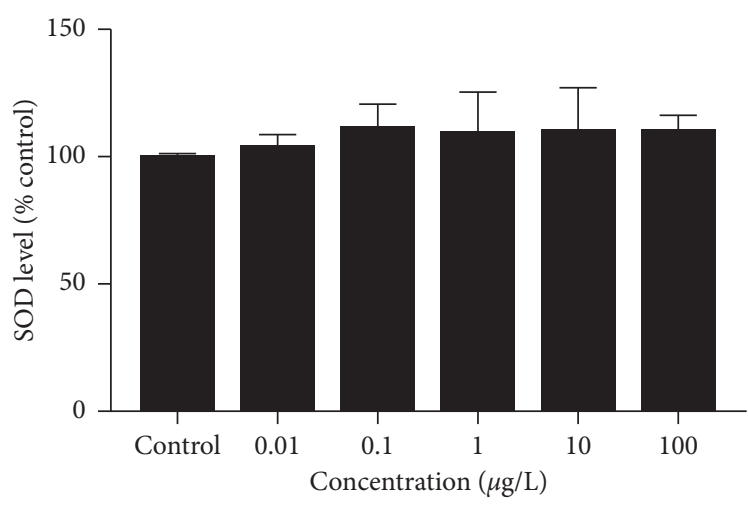

Carbendazim

(a)

(b)

FIGURE 4: Effects of C. elegans on antioxidative system exposed to carbendazim. (a) The ROS level of C. elegans after carbendazim exposure; (b) the SOD level of $C$. elegans after carbendazim exposure. Data (mean \pm SEM) are shown as the percentage value compared with the control group. The asterisks present significance between the each exposure group and control group $\left({ }^{*} p<0.05\right.$ and $\left.{ }^{* *} p<0.01\right)$.

$\beta$-tubulin, which results in impairing the segregation of chromosomes in the process of cell division [24]. The formation of microtubules by noncovalent bindings of $\alpha$ - and $\beta$-tubulin is responsible for chromosome segregation in the process of mitosis and meiosis [24]. The brood size of C. elegans significantly decreases at $0.1 \mu \mathrm{g} / \mathrm{L}$ carbendazim concentration. Carbendazim has been found to affect the reproduction systems in Japanese quails [25] and hamsters [23]. It was concluded that C. elegans lifespan was significantly decreased with carbendazim concentration of $\geq 0.01 \mu \mathrm{g} / \mathrm{L}$ based on our study. Studies have shown that carbendazim has led to infertility and developmental toxicity and manifests embryo toxicity, germ cell apoptosis, and teratogenesis in different mammalian species [17, 18, 24].

Apoptosis is a complex programmed cell death, which is a highly regulated phenomenon characterized by a series of cellular processes $[26,27]$. Many studies have presented that the production of ROS induced by oxidative stress is related to apoptotic cell death [28]. Our study found that carbendazim could induce a significant increase in the level of ROS values and a little increase in the level of SOD values. Oxidative stress caused by environmental pollution induces the increased expression of ROS and subsequently damages the antioxidant defense system [29]. SOD is responsible for the detoxification of toxic free radicals and their activities, which is used to evaluate the oxidative stress level and cellular antioxidant status $[17,24]$. Metalloenzyme SOD accelerates the transformation of endogenous cytotoxic superoxide radicals to $\mathrm{H}_{2} \mathrm{O}_{2}$, and the increase of SOD expression levels may contribute to improving the enzyme activities in order to eliminate the superoxide radicals induced by carbendazim and to prevent the occurrence of cellular dysfunction during exposure of carbendazim $[24,29]$. Higher exposure concentrations of carbendazim could cause severe oxidative stress, which subsequently destroys the balance of cell homeostasis and promote apoptosis [30]. However, carbendazim in low concentrations could still significantly damage the reproductive system according to our results.

\section{Conclusion}

As far as we know, the present study evaluated the safety of carbendazim exposed to $C$. elegans for the first time. It demonstrated that carbendazim could have a harmful effect on the locomotive behavior, development and growth, reproduction, lifespan, and antioxidant system of C. elegans. Hope that it needs to pay more attention to the application of carbendazim based on the results. In addition, the safety of carbendazim for use needs to evaluate further, especially the bioaccumulation toxicity and potential genotoxic effects.

\section{Data Availability}

All data generated or analyzed during this study are included in this article.

\section{Conflicts of Interest}

The authors declare that they have no conflicts of interest.

\section{Acknowledgments}

This research was supported by the National Natural Science Foundation of China (31501569).

\section{References}

[1] H. Li, E. Y. Zeng, and J. You, "Mitigating pesticide pollution in China requires law enforcement, farmer training, and technological innovation," Environmental Toxicology and Chemistry, vol. 33, no. 5, pp. 963-971, 2014.

[2] W. Han, Y. Tian, and X. Shen, "Human exposure to neonicotinoid insecticides and the evaluation of their potential toxicity: an overview," Chemosphere, vol. 192, pp. 59-65, 2018.

[3] U. Bajwa and K. S. Sandhu, "Effect of handling and processing on pesticide residues in food- a review," Journal of Food Science and Technology, vol. 51, no. 2, pp. 201-220, 2014.

[4] A. Farag, H. Ebrahim, R. ElMazoudy, and E. Kadous, "Developmental toxicity of fungicide carbendazim in female 
mice," Birth Defects Research Part B: Developmental and Reproductive Toxicology, vol. 92, no. 2, pp. 122-130, 2011a.

[5] W. H. Goodson III, L. Lowe, DO. Carpenter et al., "Assessing the carcinogenic potential of low-dose exposures to chemical mixtures in the environment: the challenge ahead," Carcinogenesis, vol. 36, no. Suppl 1, pp. S254-S296, 2015.

[6] A. L. G. Ferreira, S. Loureiro, and A. M. V. M. Soares, "Toxicity prediction of binary combinations of cadmium, carbendazim and low dissolved oxygen on Daphnia Magna," Aquatic Toxicology, vol. 89, no. 1, pp. 28-39, 2008.

[7] D. Liang, Y. Ren, J. Li et al., "Detection of carbendazim residues in aqueous samples by fluorescent quenching of plant esterase," Journal of Applied Spectroscopy, vol. 85, no. 3, pp. 535-542, 2018.

[8] E. P. da Costa, S. E. C. Bottrel, M. C. V. M. Starling, M. M. D. Leão, and C. C. Amorim, "Degradation of carbendazim in water via photo-fenton in raceway pond reactor: assessment of acute toxicity and transformation products," Environmental Science and Pollution Research, vol. 36, no. 5, pp. 4324-4336, 2018.

[9] F. R. G. Amrit, R. Ratnappan, S. A. Keith, and A. Ghazi, “The C. elegans lifespan assay toolkit," Methods, vol. 68, no. 3, pp. $465-475,2014$.

[10] A. K. Corsi, W. Bruce, and C. Martin, "A Transparent Window into Biology," A Primer onCaenorhabditis Elegans Genetics, vol. 200, pp. 387-407, 2015.

[11] X. Xiang, X. Zhang, X. Zhang et al., "Toxicity and multigenerational effects of bisphenol $S$ exposure to Caenorhabditis elegans on developmental, biochemical, reproductive and oxidative stress," Toxicology Research, vol. 8, no. 5, pp. 630-640, 2019.

[12] R. Farias-Pereira, J. Oshiro, K.-H. Kim, and Y. Park, "Green coffee bean extract and 5-O-caffeoylquinic acid regulate fat metabolism in Caenorhabditis elegans," Journal of Functional Foods, vol. 48, pp. 586-593, 2018.

[13] Q. Rui, Y. Zhao, Q. Wu, M. Tang, and D. Wang, "Biosafety assessment of titanium dioxide nanoparticles in acutely exposed nematode Caenorhabditis elegans with mutations of genes required for oxidative stress or stress response," Chemosphere, vol. 93, no. 10, pp. 2289-2296, 2013.

[14] X. Zhang, Y. Huang, P. R. Harvey et al., "Isolation and characterization of carbendazim-degrading Rhodococcus erythropolis djl-11," PLoS One, vol. 8, no. 10, p. e74810, 2013.

[15] T. S. Andrade, J. F. Henriques, A. Almeida et al., "Corrigendum to "Carbendazim exposure induces developmental, biochemical and behavioural disturbance in zebrafish embryos"” Aquatic Toxicology, vol. 173, p. 228, 2016.

[16] A. Ludwikowskaaga, B. Bojarski, M. Socha, H. Lutnicka, and K. B. Trzeciak, "The effect of carbendazim on embryonic Prussian carp (Carassius gibelio) development and hatching," Archives of Polish Fisheries, vol. 21, pp. 367-371, 2013.

[17] S. Singh, N. Singh, V. Kumar et al., "Toxicity, monitoring and biodegradation of the fungicide carbendazim," Environmental Chemistry Letters, vol. 14, no. 3, pp. 317-329, 2016.

[18] T. Singh, W. Li, and J. Li, "Toxicity and metabolic fate of the fungicide carbendazim in the typical freshwater diatom Navicula sp," Journal of Agricultural and Food Chemistry, vol. 67, no. 24, pp. 6683-6690, 2019.

[19] C. Nwani, M. C. Omah, N. Ivoke, H. O. Nwamba, and S. U. Ogbonna, "Biochemical, haematological and morphological variations in juvenile Clarias gariepinus exposed to Carbendazim ${ }^{\circledR}$ fungicide African," Journal of Aquatic Science, vol. 40, no. 1, pp. 63-71, 2015.
[20] K. B. Tierney, "Behavioural assessments of neurotoxic effects and neurodegeneration in Zebrafish," Biochimica et Biophysica Acta (BBA)-Molecular Basis of Disease, vol. 1812, no. 3, pp. 381-389, 20111.

[21] S.-Y. Cheng, J. L. Leonard, and P. J. Davis, "Molecular aspects of thyroid hormone actions," Endocrine Reviews, vol. 31, no. 2, pp. 139-170, 2010.

[22] D. Crump, S. Chiu, and K. L. Williams, "Bisphenol S alters embryonic viability, development, gallbladder size, and messenger RNA expression in chicken embryos exposed via egg injection," Environmental Toxicology and Chemistry, vol. 35, no. 6, pp. 1541-1549, 2016.

[23] L. Williams, J. Ostby, R. Linder, J. Goldman, and R. Cooper, "Carbendazim-induced alterations of reproductive development and function in the rat and hamster," Fundamental and Applied Toxicology, vol. 15, no. 2, pp. 281-297, 1990.

[24] E. M. Rama, S. Bortolan, M. L. Vieira, D. C. C. Gerardin, and E. G. Moreira, "Reproductive and possible hormonal effects of carbendazim," Regulatory Toxicology and Pharmacology, vol. 69, no. 3, pp. 476-486, 2014.

[25] T. A. Aire, "Short-term effects of carbendazim on the gross and microscopic features of the testes of Japanese quails (Coturnix coturnix japonica)," Anatomy and Embryology, vol. 210, no. 1, pp. 43-49, 2005.

[26] Y. Peng, R. Gan, H. Li et al., "Absorption, Metabolism, and Bioactivity of Vitexin: recent advances in understanding the efficacy of an important nutraceutical," Critical Reviews in Food Science and Nutrition, pp. 1-16, 2020.

[27] J. Ying, J. Xu, L. Shen et al., "The effect of sodium fluoride on cell apoptosis and the mechanism of human lung BEAS2B cells in vitro," Biological Trace Element Research, vol. 179, no. 1, pp. 59-69, 2017.

[28] S. A. Xu and S. Y. Shalaby, "Carbendazim-induced testicular damage and oxidative stress in albino rats: ameliorative effect of licorice aqueous extract," Toxicology and Industrial Health, vol. 30, no. 3, pp. 259-267, 2012.

[29] S. E. Owumi, S. O. Nwozo, and E. S. Najophe, "Quercetin abates induction of hepatic and renal oxidative damage, inflammation, and apoptosis in carbendazim-treated rats," Toxicology Research and Application, vol. 3, Article ID 239784731984952, 2019.

[30] I. A. Adedara, S. Vaithinathan, R. Jubendradass, P. P. Mathur, and E. O. Farombi, "Kolaviron prevents carbendazim-induced steroidogenic dysfunction and apoptosis in testes of rats," Environmental Toxicology and Pharmacology, vol. 35, no. 3, pp. 444-453, 2013. 